\title{
COVID-19 and scientific research interests and findings in epidemiology and social sciences: a systematic review
}

\author{
Bruno Enagnon Lokonon ${ }^{1}$, Marcel Gbaguidi Alia ${ }^{1}$, Romain Glèlè Kakaï ${ }^{1 *}$ \\ ${ }^{1}$ Laboratoire de Biomathématiques et d'Estimations Forestières, Université d'Abomey-Calavi, Bénin \\ *Corresponding Author: romain.glelekakai@fsa.uac.bj
}

\section{Abstract}

The emergence of COVID-19 has prompted an unprecedented scientific publication with the aim of better understanding this new disease. This study assessed the scientific impact and disciplinary priorities of the published papers on the pandemic by comparing epidemiological (EP) and social sciences (SS) research interests. Papers were identified via keywords searching using Google Scholar and Scopus databases. From an initial 1720 papers, we identified 597 relevant articles, of which 347 were for EP researches and 250 for SS studies. We extracted information, such as authors' countries, and research thematic related to EP and SS. The results revealed that most papers were authored by Asian (37.5\%), European (30.5\%) and American (19.6\%) scientists. Only $10.1 \%$ and $2.3 \%$ of authors were affiliated with African and Oceanian institutions, respectively, indicating that the regions most affected by the pandemic mainly contributed to the scientific publications. In total, 26 research themes were recorded from both EP and SS studies. There was a high significant difference among themes in both research fields $\left(\chi^{2}=1204.3, \mathrm{df}=1, p\right.$-value $\left.<0.001\right)$. EP papers mostly dealt with clinical trials $(54.5 \%)$ and diagnosis (53.3\%). These papers assessed the incidence and epidemiological characteristics of the disease (incubation period, symptomatic period, recovering or death), ${ }^{15}$ testing tests developed, drugs and vaccines used. SS papers were mainly concerned with the sociocultural analyses $(78 \%)$ and economic impact $(55.6 \%)$ of the pandemic. They mainly focused on behavioral changes induced by the pandemic and strategies developed to mitigate its impacts. This study highlights the difference between regions and gaps between scientific disciplines concerning the proposed responses to control the pandemic. It is important to 20 promote collaborative and interdisciplinary studies for health emergencies.

Keywords: SARS-Cov-2, geographical regions, research interests and findings, collaborative research 


\section{Introduction}

The novel coronavirus (SARS-Cov-2) causes COVID-19, a severe and acute respiratory syndrome discovered in Wuhan, China (Velavan and Meyer, 2020; Wu and McGoogan, 2020). By 1st March 2021, about 113,467,303 total confirmed cases and 2,520,550 deaths recorded worldwide (WHO, 2021) can be considered as proof of the ravages caused by the disease. On 11 March 2020, the World Health Organization (WHO) declared COVID-19 as a pandemic (i.e. a public health emergency of international concern), thereby underlining its global significance (WHO, 2020). This measure gave rise to numerous recommendations and guidelines on prevention and management from WHO itself and other predominantly vulnerable countries (Phua et al., 2020).

To control the pandemic, Non-Pharmaceutical Interventions (NPI), such as travel or movement restrictions, social-distancing, wearing masks and regular hand washing with water and soap have been promoted (Gilmore et al., 2020; Ngonghala et al., 2020; Kaslow et al., 2020). Meanwhile, scientists have been actively engaged in several research works to understand the dynamic of the pandemic (Cao et al., 2020; Gnanvi et al., 2020; Tovissodé et al., 2020). A 15 number of research works focused on the use of mathematical models (Taboe et al., 2020; Anastassopoulou et al., 2020; Ngonghala et al., 2020). In this category, several models have been developed to predict the course of the disease (Golinski and Spencer, 2020; Agosto and Giudici, 2020; Kosmidis and Macheras, 2020). These research works have a fundamental role in guiding public health authorities to better manage the pandemic. There were also studies related to the analysis of conditions in which vaccines and other drugs can quickly lead to a community herd immunity to curtail the pandemic (Iboi et al., 2020; Gumel et al., 2021). However, research works in social sciences (SS) have less been highlighted in the search for solutions against the pandemic (Leslie et al., 2020; Van Bavel et al., 2020). Epidemiological (EP) models and clinical treatments do not always take into account the socio-cultural behaviors of the populations facing a pandemic (Abramowitz et al., 2018). In addition, social scientists are not always able to translate their knowledge on social behaviors into epidemiologically relevant insights (Abramowitz et al., 2018). Beyond bio-medical and EP issues, the COVID-19 crisis has also social and cultural impacts (UNESCO, 2020). Decision support tools to understand and guide behavior during this crisis should also go through the social sciences. An analysis of the 30 
adaptability of society to the pandemic, a critical assessment of the solutions proposed by the political authorities, and relations between governments and populations during the pandemic are contributions that SS can provide (Leslie et al., 2020).

The research questions addressed in this study were: (1) What has been the relative productivity in scientific publications related to COVID-19 in EP and SS? (2) what are the range of thematic topics addressed by the published EP and SS papers regarding COVID-19? and (3) do the themes prioritized in both research fields converge or diverge? The objectives of the study were to (1) assess the involvement of the scientific communities in research on COVID-19 in the field of EP and SS, (2) analyze the research themes addressed by the authors in EP and SS regarding the pandemic, and (3) summarize the main findings from the selected papers in 10 both research fields.

\section{Methods}

\subsection{Search strategy and papers selection}

This study was conducted following the Systematic Reviews and Metanalyses (PRISMA) guidelines (Casals et al., 2014) (Figure 1). The search consisted of identifying all original published articles on COVID-19 using Google Scholar and Scopus databases. To find the papers, we used the following keywords: "COVID-19", "coronavirus", "SARS-CoV-2", "2019-nCoV", "n-CoV", and "Pandemic". The following fields were included separately in the search in combination with the keywords: "Epidemiology" and "social sciences". The search was restricted to published articles between 1 January 2020 and 31 December 2020. 


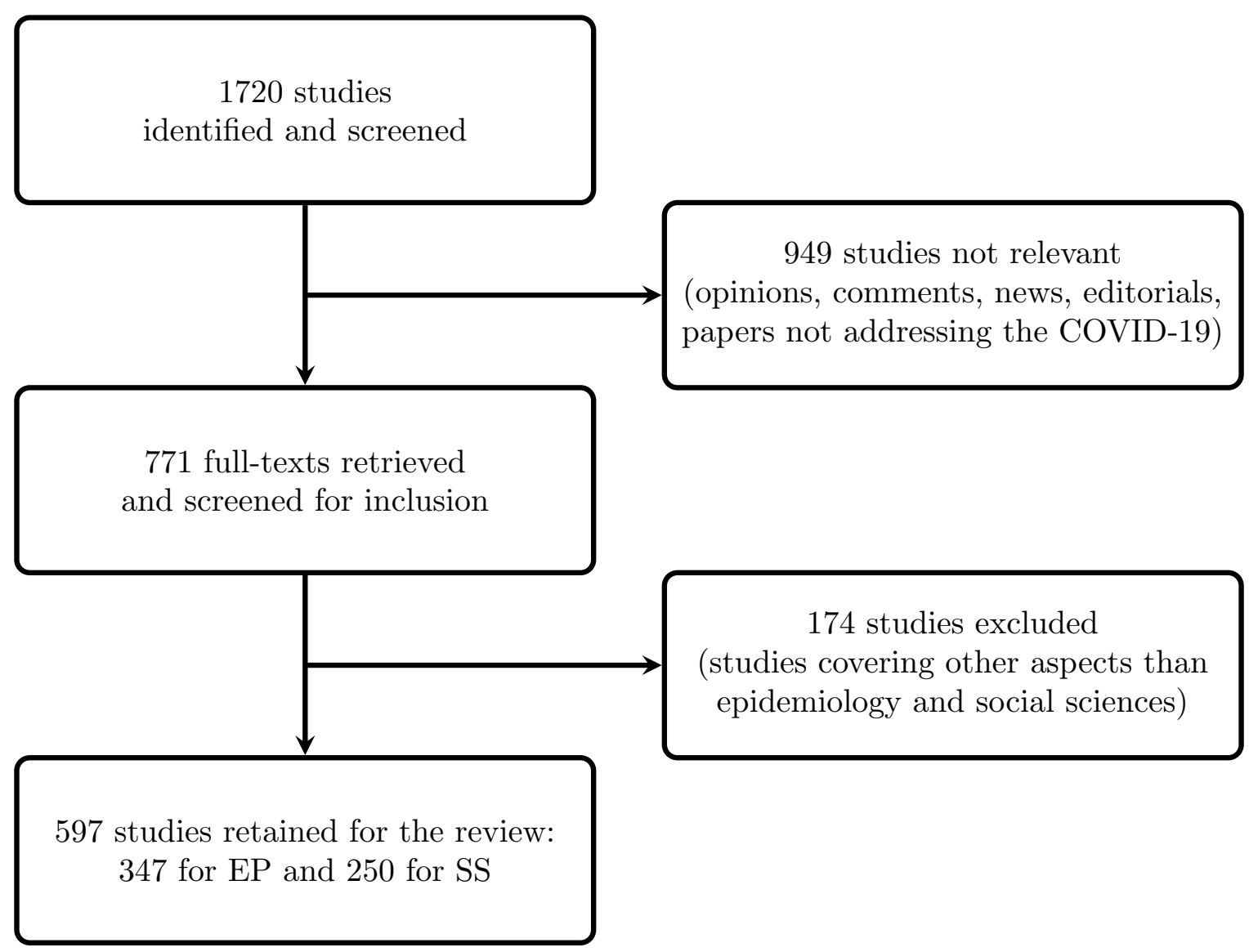

Figure 1: PRISMA flow diagram.

Two levels of screening were used. At the first level, abstracts were reviewed for the exclusion criteria. Indeed, abstracts without full-texts, proceedings, letters or commentaries, news reports and news reviews were excluded. Full-text versions were obtained for all articles accepted at this level. At the second level, distinct inclusion and exclusion criteria were applied to the 2 research fields (epidemiology and social sciences). For EP studies, papers selected included a primary collection or use of data related to COVID-19. Concerning the SS, selected articles reported SS themes linked to the coronavirus disease.

From a list of 1720 papers, 597 were finally retained with 347 from EP and 250 for SS studies (see Supplementary Material for the full list).

\subsection{Data extraction and analysis}

Data extraction was performed separately by two people following the approach used by Abramowitz et al. (2018). The two separate analyses were then merged and disagreements 
between the themes and sub-themes were reconciled. From each included papers, the following data were extracted: geographical location (country and continent) of authors, funded or unfunded status of the study and source of funding. To analyze the range of research topics addressed by the EP and SS published articles, themes and subthemes were extracted from a review of full-text articles. Each theme was coded as a binary variable $(0=$ No, $1=$ Yes $)$ when a related subtheme was mentioned or not in the full-text.

Count and relative frequencies were computed and barplots were used to describe provenance of the authors, funded or unfunded status of the study and source of funding. Moreover, proportions of themes were calculated for EP and SS and a comparison test of two proportions was performed to assess the difference between the two research fields. The analyses were conducted using $\mathrm{R}$ software, version 4.0.2 ( $\mathrm{R}$ Core Team, 2020). The main findings from the selected papers were summarized and future research directions were provided.

\section{Results}

\subsection{Characteristics of the selected papers: geographical distribution, collaboration and funding}

Most papers were authored by Asian (37.5\%), European (30.5\%) and American (19.6\%) researchers (Figure 2). Only $10.1 \%$ and $2.3 \%$ of authors were respectively affiliated with African and Oceanian institutions. In addition, most of the research studies were carried out in one geographical region $(79.3 \%$ ), whereas only $20.7 \%$ were from collaborations between geographical regions (continents). 


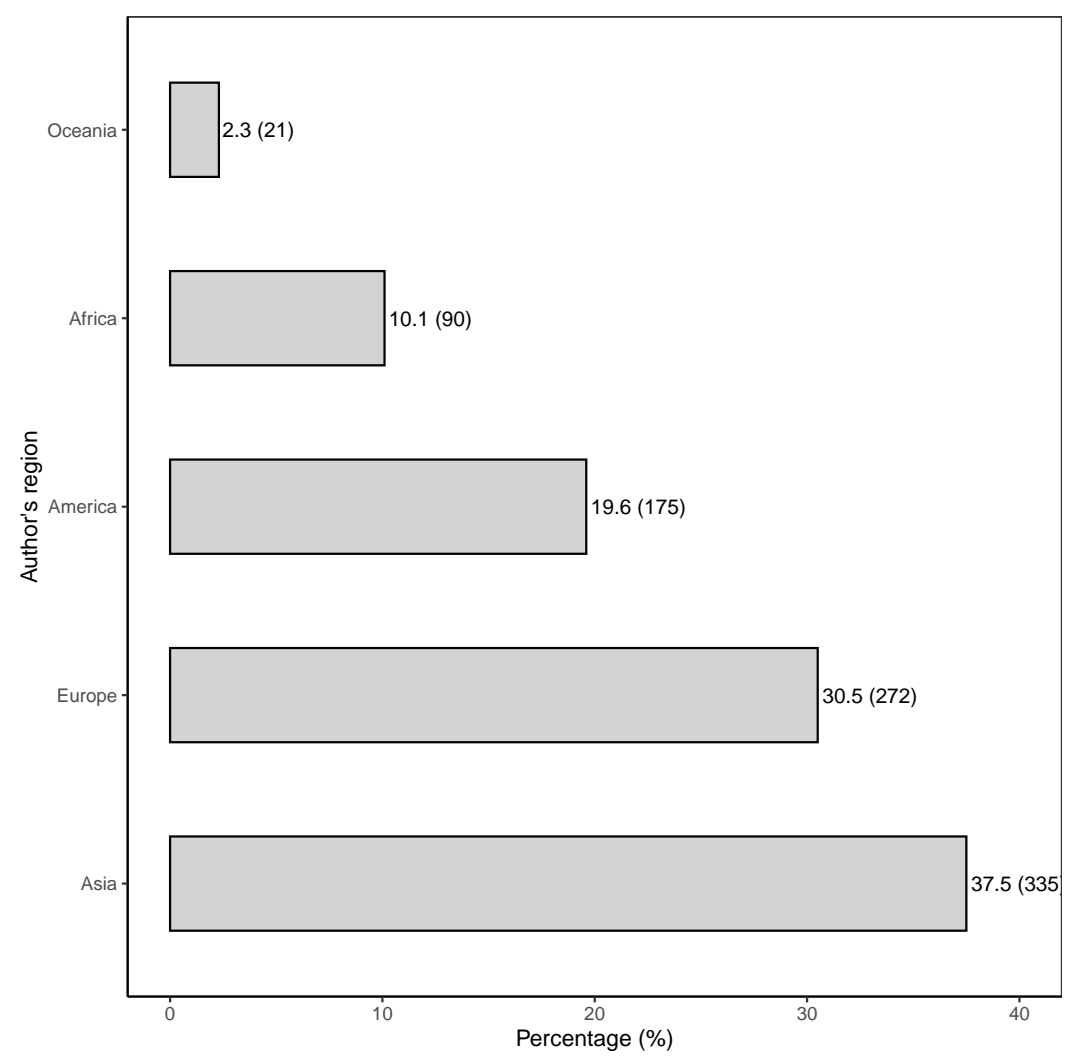

Figure 2: Distribution of authors per region.

Table 1 presents per country, the relative productivity in scientific publications related to COVID-19 in EP and SS for the most represented countries. Most of the papers came from China (15.9\%), United States (12.1\%) and Italy (7.6\%). 
Table 1: Distribution of papers per country.

\begin{tabular}{lrlr}
\hline Country & $\boldsymbol{\%}(\mathbf{n})$ & Country & $\boldsymbol{\%}(\mathbf{n})$ \\
\hline China & $15.9(142)$ & Greece & $2.1(19)$ \\
USA & $12.1(108)$ & Saudi Arabia & $2.1(19)$ \\
Italy & $7.6(68)$ & Nigeria & $1.9(17)$ \\
South Korea & $5.0(45)$ & Israel & $1.8(16)$ \\
Canada & $4.8(43)$ & South Africa & $1.7(15)$ \\
UK & $4.8(43)$ & Iran & $1.7(15)$ \\
India & $4.1(37)$ & Malaysia & $1.7(15)$ \\
Spain & $2.8(25)$ & Poland & $1.6(14)$ \\
France & $2.8(25)$ & Tanzania & $1.6(14)$ \\
Germany & $2.7(24)$ & Switzerland & $1.5(13)$ \\
Brazil & $2.7(24)$ & Egypt & $1.3(12)$ \\
Singapore & $2.7(24)$ & Cameroon & $1.2(11)$ \\
Russia & $2.6(23)$ & Kenya & $1.2(11)$ \\
Japan & $2.5(22)$ & Benin & $0.9(8)$ \\
Australia & $2.4(21)$ & Sierra Leone & $0.2(2)$ \\
Belgium & $2.0(18)$ & & \\
\hline
\end{tabular}

The majority of the selected papers $(66.8 \%)$ were not financially supported $(28.8 \%$ gave no information about funding and 38.0\% stated that they did not receive any funding) (Figure 3). In total, $13.4 \%$ of the selected papers were funded by European, $12.4 \%$ by Asian, $6.4 \%$ by American and $0.5 \%$ by African and Oceanian institutions, respectively. Moreover, it is noted that, European funding mainly supported European authors (82.7\%) and Asian funding mainly $\quad 5$ supported Asian authors (78.5\%). 


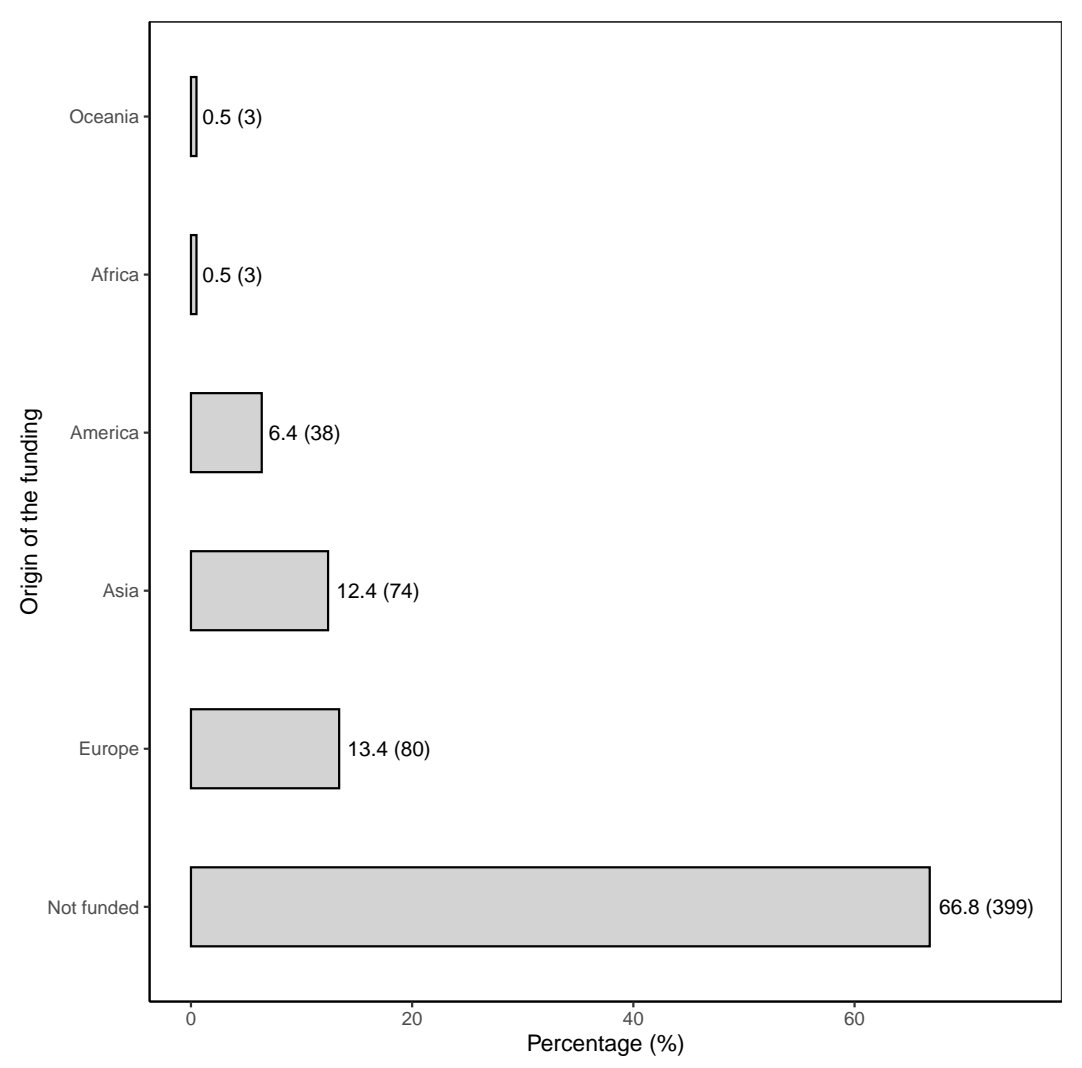

Figure 3: Breakdown of funding granted to research works on COVID-19.

\subsection{Relative importance of themes considered}

A total of 26 themes were identified with 350 subthemes. Table 2 presents the number (n) and percentage (\%) of papers per themes for EP and SS, respectively.

EP papers prioritized studies related clinical trials (54.5\%), diagnosis of COVID-19 (53.3\%) and other EP themes including incidence, mortality and characterizing outbreaks $(37.4 \%)$. These proportions were significantly higher $($ Prob $<0.05)$ than those for SS (Table 2). SS papers prioritized Sociocultural themes $(78 \%)$ and the economic impact of the pandemic (55.6\%). Other themes related to prevention and response to the pandemic were considered. These themes were: ethics (53.1\%), funerary practices and burials (53.1\%) and political issues (51.8\%). All these proportions were significantly higher $($ Prob $<0.05)$ than those for EP papers (Table 2). 10 Finally, the two research fields showed similar priority (Prob $>0.05$ ) for the themes related to clinical characteristics, health systems, comparison of COVID-19 with past disease outbreaks and modelling (Table 2). 
Table 2: Themes and proportions of papers for EP and SS.

\begin{tabular}{lrrr}
\hline Themes & EP [\%(n)] & SS [\%(n)] & Prob \\
\hline Age groups/vulnerable populations & $13.2(46)$ & $23.2(58)$ & 0.002 \\
Alternative health practices & $1.5(5)$ & $46.9(117)$ & $<0.001$ \\
Clinical characteristics & $40.9(142)$ & $34.4(86)$ & 0.125 \\
Clinical trials & $54.5(189)$ & $42.4(86)$ & 0.005 \\
Community engagement & $19.4(67)$ & $45.6(114)$ & $<0.001$ \\
Diagnosis & $53.3(185)$ & $9.5(24)$ & $<0.001$ \\
Economic impact & $7.8(27)$ & $55.6(139)$ & $<0.001$ \\
Epidemiology themes & $37.4(130)$ & $22.8(57)$ & 0.005 \\
Ethics & $10.5(36)$ & $53.1(133)$ & $<0.001$ \\
Funerals/burials & $1.5(5)$ & $53.1(133)$ & $<0.001$ \\
Health communications strategies & $6.9(24)$ & $39.4(99)$ & $<0.001$ \\
Health systems & $25.7(89)$ & $39.4(99)$ & 0.060 \\
Mobility & $10.5(36)$ & $50.6(127)$ & $<0.001$ \\
Modelling & $33.4(116)$ & $27.2(68)$ & 0.124 \\
Other diseases addressed/compared & $32(111)$ & $40.6(102)$ & 0.235 \\
Political themes & $5.1(18)$ & $51.8(130)$ & $<0.001$ \\
Risk factors & $14(49)$ & $38.1(96)$ & $<0.001$ \\
Psychosocial & $7.8(27)$ & $49.3(124)$ & $<0.001$ \\
Public health response & $8.7(30)$ & $43.1(108)$ & $<0.001$ \\
Post-Covid & $6(21)$ & $43.1(108)$ & $<0.001$ \\
Rumors, myths and misinformation & $0.6(2)$ & $9.5(24)$ & 0.004 \\
Sociocultural themes & $26.6(92)$ & $78(195)$ & 0.002 \\
Sensitivity-specificity & $6(21)$ & $44.4(111)$ & $<0.001$ \\
Transportation & $6(21)$ & $40.6(102)$ & $<0.001$ \\
Survivors reintegration & $9.6(33)$ & $38.1(96)$ & $<0.001$ \\
Transmission & $18.5(64)$ & $41.9(105)$ & 0.001 \\
\hline & & & \\
& & & \\
& & &
\end{tabular}




\subsection{Summary of main findings on COVID-19}

Key results obtained from the reviewed papers are presented below.

\section{Diagnosis}

To detect COVID-19, several tests have been developed according to the reviewed papers. Nucleic acid amplification tests such as real-time RT-PCR are the most widely used and recommended test to confirm infection with SARS-CoV-2 (Wang et al., 2020). Rapid diagnostic tests detecting viral proteins with the potential to speed up and simplify the detection of active SARS-CoV-2 infection are also used (Zhang et al., 2020). Studies have shown that in most cases, SARS-CoV-2 becomes detectable in the upper respiratory tract around 1 to 3 days before the symptoms onset and for several days or weeks after the symptomatic period (To 10 et al., 2020). The average time between exposure to SARS-CoV-2 and symptoms onset (the incubation period) is 5 to 6 days, but can vary from 1 to 14 days and it is estimated that in $17 \%$ to $25 \%$ of cases, the virus may be detectable without developing symptoms (Kronbichler et al., 2020).

\section{Clinical trials}

Several papers have presented results from clinical trials assessing the efficacy of drugs. The effect of drugs on 3 important outcomes in COVID-19 patients (mortality, need for assisted ventilation and duration of hospital stay) were assessed in most studies (Barnabas et al., 2020). In the cases of remdesivir, hydroxychloroquine, lopinavir/ritonavir and interferon, little or no reduction in the mortality, need for assisted ventilation and duration of hospital stay were observed (Barnabas et al., 2020). However, Spinner et al. (2020) stated that patients randomized to a 5-day course of remdesivir had a statistically significant difference in clinical status compared with the standard care, but the difference was of uncertain clinical importance. Moreover, there are many official vaccine projects subjected to clinical trials (Polack et al., 2020). The first authorized and recommended vaccines to prevent COVID-19 are Pfizer-BioNTech 2 Moderna's COVID-19 vaccines (Anderson et al., 2020; Polack et al., 2020). According to the reviewed papers, both vaccines have shown approximately 95\% efficacy in preventing symptomatic COVID-19 infections in phase 3 trials, without significant safety concerns that might 
hinder authorization (Polack et al., 2020).

\section{Age groups/vulnerable populations}

Several studies found a significant impact of age on the clinical characteristics and outcomes of COVID-19 patients (Zhao et al., 2020; Zhou et al., 2020; Verity et al., 2020; Zhang et al., 2020). The symptoms of the aged patients were more atypical than those of the young patients and were characterized by more comorbidities (Zhao et al., 2020). Moreover, older patients had more severe inflammation on admission and during hospitalization, they received oxygen therapy and experienced more complications with a significantly higher mortality rate (Verity et al., 2020; Zhang et al., 2020; Zhao et al., 2020). However, more recent studies showed higher rates of severe forms of COVID-19 in younger populations due to the mutations of the virus (Garvin et al., 2020).

\section{Modelling}

Modelling techniques have been profusely used in the scientific papers as a tool to assess the COVID-19 disease transmission dynamics and to predict its future course. The most used models were compartmental and statistical models (Tovissodé et al., 2020; Anastassopoulou et al., 2020; Agosto and Giudici, 2020; Taboe et al., 2020). The compartmental model divided the population into different sub-populations, such as Susceptible, Exposed, Infected, Quarantined, Recovered, and Dead (Ngonghala et al., 2020). Classical and improved versions of the compartmental models have been considered in many studies (Cao et al., 2020; Ngonghala et al., 2020; Taboe et al., 2020). Statistical models included growth models, spatial models, 20 time series models, Poisson models and their alternatives (Agosto and Giudici, 2020; Tovissodé et al., 2020). Another class of models widely used are machine learning models (Gupta et al., 2020; Farooq and Bazaz, 2020). These models have contributed among others to (i) assess the impact of control interventions; (ii) generate short and long-term forecasts; (ii) determine epidemic peak time and size, epidemic size and duration (Agosto and Giudici, 2020; Tovissodé ${ }_{25}$ et al., 2020). 


\section{Social and cultural analyses}

Findings of several studies revealed that communities responded to the pandemic in various ways (Sutin et al., 2020; Croll et al., 2020; Dong et al., 2020; Lesser and Nienhuis, 2020). Sociocultural factors, such as behaviors, beliefs and practices, affect the responses of the communities (Duan et al., 2020; Jeffrey, 2020). For instance, while in some countries control and preventive measures, such as lockdown, closure of non-essential establishments and businesses were successfully respected, in other countries these measures were not or partially accepted (Doogan et al., 2020). Other papers have shown several negative psychological effects of social isolation of COVID-19 patients, such as high levels of anxiety, stress, or even the presence of depressive symptoms that can persist after the pandemic (Antunes et al., 2020; Duan et al., 10 2020). Antunes et al. (2020) found that women presented higher levels of state anxiety and trait anxiety when compared to men. An age-related variation was also found, among the youngest (18-34 years) groups showing higher levels of trait anxiety (Antunes et al., 2020).

\section{Economics and political issues}

COVID-19 has not only caused a health crisis but also a general slowdown in economic activities, 15 especially for small and medium-sized enterprises (Kim et al., 2020; Song et al., 2020; Xie et al., 2020). It has serious impacts on trade, as well as on public and international policies (Bruns et al., 2020; Miller, 2020; Motta Zanin et al., 2020). The management of the pandemic has given rise to many doubts about the ability of leaders and administrative systems to manage the crisis (Cohen et al., 2020; Shatri et al., 2020). Some countries have undertaken measures to support workers who lost their jobs during the pandemic and to offer help to vulnerable people (Bruns et al., 2020; Meisner et al., 2020; Blustein and Guarino, 2020; Carroll et al., 2020). In addition, policy makers have not only been faced with the arduous task of finding viable solutions to respond effectively to the health crisis but also to the economic emergency to support vulnerable businesses and maintain financial stability (Cohen et al., 2020; Jeffrey, 2020; 2 Li et al., 2020; Miller, 2020). However, despite its negative impacts on economy, the pandemic is also seen as an opportunity in some studies. Indeed, the current pandemic situation can increase the development of newer technologies (Okyere et al., 2020). These innovations may contribute to efficient ways and means of productions and low-cost productions (Karunathilake, 2020). 
Following the onset of the pandemic, several countries faced serious ethical challenges (Jeffrey, 2020). These include, but are not limited to: resources allocation, rights and duties of workers (Jeffrey, 2020; Miller, 2020; Cohen et al., 2020; Carroll et al., 2020; Ogden, 2020; Shatri et al., 2020; Sorokowski et al., 2020). These challenges were complicated by a health system and a socio-economic and cultural context of each country (Dong et al., 2020; Lesser and Nienhuis, 5 2020).

\subsection{COVID-19 Research Perspectives}

Although efforts are being made by the scientific communities worldwide to understand and fight against COVID-19, many unknowns remain regarding this pandemic. Some research subjects raised in the reviewed papers that must be addressed in future studies are presented below.

- Use of Artificial Intelligence (AI) techniques to understand COVID-19 dynamic. AI methods have been moderately used in the battle against COVID-19. AI can help to address many issues posed by COVID-19. For instance, mathematical foundations of AI can be used for real-time spread tracking, early warning and alerts for particular geographical 15 locations and to provide accurate forecasting.

- Modelling optimal vaccine allocation strategies within and between countries to maximize health under constraints on dose supply.

- Modelling the public health impact of the COVID-19 vaccines.

- Assessing social, psychological, and economical impacts of COVID-19 on low-income coun- ${ }_{20}$ tries.

- Assessing positive effects of COVID-19 on the environment and natural ecosystems.

- Incorporation of social behavior during the COVID-19 pandemic into mathematical models.

- Modelling the impact of environmental factors on the spread of COVID-19 according to ${ }^{25}$ the main climatic zones of the world.

- Assessing the impacts of COVID-19 on small and medium-sized businesses in Africa.

- Assessing the determinants of COVID-19 vaccines acceptance in African countries. 


\section{Discussion}

\subsection{Scientific response to the COVID-19 pandemic}

In response to the COVID-19 pandemic, scientific research is emerging at an unprecedented rate. Between 1 January and 30 April 2020, more than 4,000 publications on COVID-19 were reported on PubMed, with an average of about 33 publications everyday (Sarkis et al., 2020). It would have taken 24 months to reach the same number of articles during the 2009 H1N1 Influenza pandemic (Sarkis et al., 2020). Between 1 January and 30 November 2020, more than 38,000 Web of Science and 78,000 PubMed articles on COVID-19 were identified (Shapira, 2020). Behind this abundant scientific publication, our results revealed disparities between regions. There is a correlation between the regions and countries most affected by the pandemic and the scientific contribution. The regions with the highest number of publications and authors were Asia, Europe, and America while Africa and Oceania had lower published scientific papers. The same trend was observed for research funding and regional collaborations. These differences between regions may have several explanations. First, the most affected countries are also the richest in the world. Consequently, authorities and research funding institutions quickly understood the issues and provided research institutions with funds. Thus, the differences may be due to the influence of rapidly available added COVID-19 research funding (Shapira, 2020). Second, COVID-19 has also changed the traditional way of working. A new way of working has been clearly observed thanks to the increased use of new technologies, which requires expertise (IAU, 2020). Third, in some regions, there are not enough resources and equipment to conduct some kinds of studies as they were not enough prepared for this unexpected situation (Abramowitz et al., 2018). Moreover, due to the border closure for several months, researchers who should have traveled to conduct their research in equipped laboratories have been blocked. This prevented several experiments which should be performed.

These results call for strengthening regional collaboration. COVID-19 is completely a new ${ }^{25}$ situation, which invites reconsidering the existing forms of collaborations between regions. The lessons learned from previous epidemics, like Ebola or Influenza can guide. In addition, the creation of new research networks between different regions can promote exchanges of knowledge and also pooling resources and providing financial support. By funding infrastructure and research projects in the poorest regions like Africa, researchers can gain more autonomy. Skills 30 
will be gained and long-term collaborations between regions and sub-regions can be effective. This will help smooth out the knowledge differences between regions in order to be better prepared for future pandemics.

\subsection{Epidemiological and social sciences research findings}

Our research showed that the two investigated disciplines (EP and SS) have approached the 5 pandemic in different ways and on different themes. While EP addressed themes related to clinical trials, diagnosis, incidence, mortality and outbreaks, SS prioritized sociocultural themes and economic impacts. Abramowitz et al. (2018) obtained similar results from their study on Ebola. They pointed out that the approaches used by epidemiological, social and behavioral sciences often seemed diametrically opposed. They found that epidemiology is often based on population data (eg, age, sex) to make general inferences without incorporating local insights (eg, cultural practices, traditional structures) while behavioral sciences used small samples to make sweeping inferences (Abramowitz et al., 2018).

There is a need to combine the two disciplines for more effective responses to COVID-19 or other future pandemics (Moon et al., 2015). Efforts must be made to develop new approaches for interdisciplinary research. Data collection systems integrating both EP and SS variables need to be developed. Where as much of the literature in the epidemic space situates social scientists as cultural brokers (Leslie et al., 2020), this study shows that no area of research should be overlooked when faced with a pandemic, like COVID-19. Knowledge and experiences of specialists from other fields, such as economy and finance, environment or politics need to ${ }^{20}$ be integrated through interdisciplinary cooperation and setting up of collaborative projects.

\section{Conclusion}

This study highlights the disparities between regions of the world when dealing with a health emergency, such as COVID-19. It also underlines the gaps between scientific disciplines concerning the proposed responses to control the pandemic. Based on these results, creation of ${ }_{25}$ international cooperation and collaboration networks between national research centres of infectious diseases is required for an efficient and global response to pandemics. The objectives of these networks will be to prepare sub-regional and national research centres to equip themselves 
in resources and skills to effectively respond to present and future pandemics. We also suggest the establishment of new interdisciplinary and integrated research mechanisms and strategies in the sub-regional and national research centres. All experts who can bring relevant local contextual, medical, epidemiological, environmental and political information on global health emergencies must be involved.

\section{References}

Abramowitz, S. A., Hipgrave, D. B., Witchard, A., and Heymann, D. L. (2018). Lessons From the West Africa Ebola Epidemic: A Systematic Review of Epidemiological and Social and Behavioral Science Research Priorities. The Journal of infectious diseases 218, 1730-1738. doi:10.1093/infdis/jiy387

Agosto, A. and Giudici, P. (2020). A poisson autoregressive model to understand COVID-19 contagion dynamics. Risks 8, 77. doi:10.3390/risks8030077

Anastassopoulou, C., Russo, L., Tsakris, A., and Siettos, C. (2020). Data-based analysis, modelling and forecasting of the COVID-19 outbreak. PloS one 15, e0230405. doi:10.1371/ journal.pone.0230405

Anderson, E. J., Rouphael, N. G., Widge, A. T., Jackson, L. A., Roberts, P. C., Makhene, M., et al. (2020). Safety and immunogenicity of SARS-CoV-2 mRNA-1273 vaccine in older adults. New England Journal of Medicine 383, 2427-2438. doi:10.1056/NEJMoa2028436

Antunes, R., Frontini, R., Amaro, N., Salvador, R., Matos, R., Morouço, P., et al. (2020). Exploring lifestyle habits, physical activity, anxiety and basic psychological needs in a sample 20 of Portuguese adults during COVID-19. International journal of environmental research and public health 17, 4360. doi:10.3390/ijerph17124360

Barnabas, R. V., Brown, E. R., Bershteyn, A., Stankiewicz Karita, H. C., Johnston, C., Thorpe, L. E., et al. (2020). Hydroxychloroquine as Postexposure Prophylaxis to Prevent Severe Acute Respiratory Syndrome Coronavirus 2 Infection: A Randomized Trial. Annals of internal ${ }_{25}$ medicine 174, 344-352. doi:10.7326/M20-6519

Blustein, D. L. and Guarino, P. A. (2020). Work and unemployment in the time of COVID-19: the existential experience of loss and fear. Journal of Humanistic Psychology 60, 702-709. doi:10.1177/0022167820934229 
Bruns, D. P., Kraguljac, N. V., and Bruns, T. R. (2020). COVID-19: Facts, Cultural Considerations, and Risk of Stigmatization. Journal of Transcultural Nursing 31, 326-332. doi: $10.1177 / 1043659620917724$

Cao, J., Jiang, X., Zhao, B., et al. (2020). Mathematical modeling and epidemic prediction of COVID-19 and its significance to epidemic prevention and control measures. Journal of ${ }_{5}$ Biomedical Research and Innovation 1, 1-19

Carroll, N., Sadowski, A., Laila, A., Hruska, V., Nixon, M., Ma, D. W., et al. (2020). The impact of COVID-19 on health behavior, stress, financial and food security among middle to high income Canadian families with young children. Nutrients 12, 2352. doi:10.3390/nu12082352

Casals, M., Girabent-Farres, M., and Carrasco, J. L. (2014). Methodological Quality and Reporting of Generalized Linear Mixed Models in Clinical Medicine (2000-2012): A Systematic Review. PloS one 9, e112653. doi:10.1371/journal.pone.0112653

Cohen, A. K., Hoyt, L. T., and Dull, B. (2020). A descriptive study of COVID-19-related experiences and perspectives of a national sample of college students in spring 2020. Journal of Adolescent Health 67, 369-375. doi:10.1016/j.jadohealth.2020.06.009

Croll, L., Kurzweil, A., Hasanaj, L., Serrano, L., Balcer, L. J., and Galetta, S. L. (2020). The psychosocial implications of COVID-19 for a neurology program in a pandemic epicenter. Journal of the neurological sciences 416, 1-6. doi:10.1016/j.jns.2020.117034

Dong, Z.-Q., Ma, J., Hao, Y.-N., Shen, X.-L., Liu, F., Gao, Y., et al. (2020). The social psychological impact of the COVID-19 pandemic on medical staff in China: A cross-sectional study. European Psychiatry 63, 1-8. doi:10.1192/j.eurpsy.2020.59

Doogan, C., Buntine, W., Linger, H., and Brunt, S. (2020). Public perceptions and attitudes toward COVID-19 nonpharmaceutical interventions across six countries: A topic modeling analysis of Twitter data. Journal of medical Internet research 22, e21419. doi:10.2196/21419

Duan, L., Shao, X., Wang, Y., Huang, Y., Miao, J., Yang, X., et al. (2020). An investigation of ${ }^{25}$ mental health status of children and adolescents in china during the outbreak of COVID-19. Journal of affective disorders 275, 112-118. doi:10.1016/j.jad.2020.06.029

Farooq, J. and Bazaz, M. A. (2020). A deep learning algorithm for modeling and forecasting of COVID-19 in five worst affected states of India. Alexandria Engineering Journal 60, 587-596. doi:10.1016/j.aej.2020.09.037

Garvin, M. R., Prates, E. T., Pavicic, M., Jones, P., Amos, B. K., Geiger, A., et al. (2020). 
Potentially adaptive SARS-CoV-2 mutations discovered with novel spatiotemporal and explainable AI models. Genome biology 21, 1-26. doi:10.1186/s13059-020-02191-0

Gilmore, B., Ndejjo, R., Tchetchia, A., de Claro, V., Mago, E., Lopes, C., et al. (2020). Community engagement for COVID-19 prevention and control: a rapid evidence synthesis. BMJ global health 5, e003188. doi:10.1136/bmjgh-2020-003188

Gnanvi, J., Salako, V. K., Kotanmi, B., and Kakaï, R. G. (2020). On the reliability of predictions on COVID-19 dynamics: a systematic and critical review of modelling techniques. Infectious Disease Modelling , 258-272doi:10.1101/2020.09.10.20192328

Golinski, A. and Spencer, P. D. (2020). Modeling the COVID-19 Epidemic using Time Series Econometrics. medRxiv doi:10.1101/2020.06.01.20118612

Gumel, A. B., Iboi, E. A., Ngonghala, C. N., and Elbasha, E. H. (2021). A primer on using mathematics to understand COVID-19 dynamics: Modeling, analysis and simulations. Infectious Disease Modelling 6, 148-168. doi:10.1016/j.idm.2020.11.005

Gupta, R., Pandey, G., Chaudhary, P., and Pal, S. K. (2020). Machine learning models for government to predict COVID-19 outbreak. Digital Government: Research and Practice 1, 15 1-6. doi:10.1145/3411761

IAU (2020). Regional / National Perspectives on the Impact of COVID-19 on Higher Education , $40 \mathrm{p}$

Iboi, E. A., Ngonghala, C. N., and Gumel, A. B. (2020). Will an imperfect vaccine curtail the COVID-19 pandemic in the US? Infectious Disease Modelling 5, 510-524. doi:10.1016/j.idm. 20 2020.07.006

Jeffrey, D. I. (2020). Relational ethical approaches to the COVID-19 pandemic. Journal of medical ethics 46, 495-498. doi:10.1136/medethics-2020-106264

Karunathilake, K. (2020). Positive and negative impacts of COVID-19, an analysis with special reference to challenges on the supply chain in South Asian countries. Journal of Social and ${ }_{2}$ Economic Development , 1-14doi:10.1007/s40847-020-00107-z

Kaslow, N. J., Friis-Healy, E. A., Cattie, J. E., Cook, S. C., Crowell, A. L., Cullum, K. A., et al. (2020). Flattening the emotional distress curve: A behavioral health pandemic response strategy for COVID-19. American Psychologist 75, 875-886. doi:10.1037/amp0000694

Kim, Y.-J., Cho, J.-H., Kim, E., et al. (2020). Differences in sense of belonging, pride, and 30 mental health in the Daegu Metropolitan Region due to COVID-19: Comparison between the 
presence and absence of National Disaster Relief Fund. International journal of environmental research and public health 17, 4910. doi:10.3390/ijerph17134910

Kosmidis, K. and Macheras, P. (2020). A fractal kinetics SI model can explain the dynamics of COVID-19 epidemics. PloS one 15, e0237304. doi:10.1371/journal.pone.0237304

Kronbichler, A., Kresse, D., Yoon, S., Lee, K. H., Effenberger, M., and Shin, J. I. (2020). Asymptomatic patients as a source of COVID-19 infections: A systematic review and metaanalysis. International journal of infectious diseases 98, 180-186. doi:10.1016/j.ijid.2020.06. 052

Leslie, M., Fadaak, R., Davies, J., Blaak, J., Forest, P., Green, L., et al. (2020). Integrating the social sciences into the COVID-19 response in Alberta, Canada. BMJ Global Health 5, 10 e002672. doi:10.1136/bmjgh-2020-002672

Lesser, I. A. and Nienhuis, C. P. (2020). The impact of COVID-19 on physical activity behavior and well-being of Canadians. International journal of environmental research and public health 17, 1-12. doi:10.3390/ijerph17113899

Li, S., Wang, Y., Xue, J., Zhao, N., and Zhu, T. (2020). The impact of COVID-19 epidemic declaration on psychological consequences: a study on active Weibo users. International journal of environmental research and public health 17, 2032. doi:10.3390/ijerph17062032

Meisner, B. A., Boscart, V., Gaudreau, P., Stolee, P., Ebert, P., Heyer, M., et al. (2020). Interdisciplinary and collaborative approaches needed to determine impact of COVID-19 on older adults and aging: CAG/ACG and CJA/RCV joint statement. Canadian Journal on 20 Aging/La Revue canadienne du vieillissement 39, 333-343. doi:10.1017/S0714980820000203

Miller, E. A. (2020). Protecting and improving the lives of older adults in the COVID-19 Era. Journal of Aging \& Social Policy 32, 297-309. doi:10.1080/08959420.2020.1780104

Moon, S., Sridhar, D., Pate, M. A., Jha, A. K., Clinton, C., Delaunay, S., et al. (2015). Will Ebola change the game? Ten essential reforms before the next pandemic. The report of the ${ }^{25}$ Harvard-LSHTM Independent Panel on the Global Response to Ebola. The Lancet 386, 2204-2221. doi:10.1016/S0140-6736(15)00946-0

Motta Zanin, G., Gentile, E., Parisi, A., and Spasiano, D. (2020). A preliminary evaluation of the public risk perception related to the COVID-19 health emergency in Italy. International journal of environmental research and public health 17, 3024. doi:10.3390/ijerph17093024

Ngonghala, C. N., Iboi, E., Eikenberry, S., Scotch, M., MacIntyre, C. R., Bonds, M. H., 
et al. (2020). Mathematical assessment of the impact of non-pharmaceutical interventions on curtailing the 2019 novel Coronavirus. Mathematical biosciences 325, 108364. doi: 10.1016/j.mbs.2020.108364

Ogden, R. S. (2020). The passage of time during the UK Covid-19 lockdown. Plos one 15, e0235871. doi:10.1371/journal.pone.0235871

Okyere, M. A., Forson, R., and Essel-Gaisey, F. (2020). Positive externalities of an epidemic: The case of the coronavirus (COVID-19) in China. Journal of medical virology 92, 1376-1379. doi:10.1002/jmv.25830

Phua, J., Weng, L., Ling, L., Egi, M., Lim, C.-M., Divatia, J. V., et al. (2020). Intensive care management of coronavirus disease 2019 (COVID-19): challenges and recommendations. The 10 Lancet Respiratory Medicine 8, 506-517. doi:10.1016/S2213-2600(20)30161-2

Polack, F. P., Thomas, S. J., Kitchin, N., Absalon, J., Gurtman, A., Lockhart, S., et al. (2020). Safety and efficacy of the BNT162b2 mRNA Covid-19 vaccine. New England Journal of Medicine 383, 2603-2615. doi:10.1056/NEJMoa2034577

R Core Team (2020). R: A Language and Environment for Statistical Computing. R Foundation 15 for Statistical Computing, Vienna, Austria

Sarkis, J., Assaf, J., and Sarkis, P. (2020). Publications during the COVID-19 pandemic: Between quantity and quality. La Presse Médicale Formation 1, 332-336. doi:10.1016/j. lpmfor.2020.07.014

Shapira, P. (2020). Scientific publications and COVID-19 "research pivots" during the pandemic: 20 An initial bibliometric analysis. bioRxiv , 1-28doi:10.1101/2020.12.06.413682

Shatri, H., Faisal, E., and Putranto, R. (2020). Mass Panic Disaster Management in COVID-19 Pandemic. Acta Medica Indonesiana 52, 179-184

Song, L., Wang, Y., Li, Z., Yang, Y., and Li, H. (2020). Mental health and work attitudes among people resuming work during the COVID-19 pandemic: A cross-sectional study in 25 China. International journal of environmental research and public health 17, 5059. doi: 10.3390/ijerph17145059

Sorokowski, P., Groyecka, A., Kowal, M., Sorokowska, A., Białek, M., Lebuda, I., et al. (2020). Can information about pandemics increase negative attitudes toward foreign groups? A case of COVID-19 outbreak. Sustainability 12, 4912. doi:10.3390/su12124912

Spinner, C. D., Gottlieb, R. L., Criner, G. J., López, J. R. A., Cattelan, A. M., Viladomiu, 
A. S., et al. (2020). Effect of remdesivir vs standard care on clinical status at 11 days in patients with moderate COVID-19: a randomized clinical trial. Jama 324, 1048-1057. doi:10.1001/jama.2020.16349

Sutin, A. R., Luchetti, M., Aschwanden, D., Lee, J. H., Sesker, A. A., Strickhouser, J. E., et al. (2020). Change in five-factor model personality traits during the acute phase of the coronavirus pandemic. PloS one 15, e0237056. doi:10.1371/journal.pone.0237056

Taboe, H. B., Salako, K. V., Tison, J. M., Ngonghala, C. N., and Kakaï, R. G. (2020). Predicting COVID-19 spread in the face of control measures in West Africa. Mathematical biosciences 328, 108431. doi:10.1016/j.mbs.2020.108431

To, K. K.-W., Tsang, O. T.-Y., Leung, W.-S., Tam, A. R., Wu, T.-C., Lung, D. C., et al. ${ }_{10}$ (2020). Temporal profiles of viral load in posterior oropharyngeal saliva samples and serum antibody responses during infection by SARS-CoV-2: an observational cohort study. The Lancet Infectious Diseases 20, 565-574. doi:10.1016/S1473-3099(20)30196-1

Tovissodé, C. F., Lokonon, B. E., and Glèlè Kakaï, R. (2020). On the use of growth models to understand epidemic outbreaks with application to COVID-19 data. Plos one 15, e0240578. ${ }^{15}$ doi:10.1371/journal.pone.0240578

UNESCO (2020). Socio-Economic and Cultural Impacts of COVID-19 on Africa, UNESCO Responses , $15 \mathrm{p}$

Van Bavel, J. J., Baicker, K., Boggio, P. S., Capraro, V., Cichocka, A., Cikara, M., et al. (2020). Using social and behavioural science to support COVID-19 pandemic response. Nature human 20 behaviour 4, 460-471. doi:10.1038/s41562-020-0884-z

Velavan, T. P. and Meyer, C. G. (2020). The COVID-19 epidemic. Tropical medicine \& international health 25, 278-280. doi:10.1111/tmi.13383

Verity, R., Okell, L. C., Dorigatti, I., Winskill, P., Whittaker, C., Imai, N., et al. (2020). Estimates of the severity of coronavirus disease 2019: a model-based analysis. The Lancet ${ }^{25}$ infectious diseases 20, 669-677. doi:10.1016/S1473-3099(20)30243-7

Wang, Y., Zhang, L., Sang, L., Ye, F., Ruan, S., Zhong, B., et al. (2020). Kinetics of viral load and antibody response in relation to COVID-19 severity. The Journal of clinical investigation 130. doi:10.1172/JCI138759

WHO (2020). Coronavirus disease 2019 (COVID-19): situation report, 208 , 16p

WHO (2021). Weekly Operational Update on COVID-19 of 1 March 2021, 16p. 
Wu, Z. and McGoogan, J. M. (2020). Characteristics of and important lessons from the coronavirus disease 2019 (COVID-19) outbreak in China: summary of a report of 72,314 cases from the Chinese Center for Disease Control and Prevention. Jama 323, 1239-1242. doi: 10.1001/jama.2020.2648

Xie, W., Campbell, S., and Zhang, W. (2020). Working memory capacity predicts individual differences in social-distancing compliance during the COVID-19 pandemic in the United States. Proceedings of the National Academy of Sciences 117, 17667-17674. doi:10.1073/ pnas. 2008868117

Zhang, G., Hu, C., Luo, L., Fang, F., Chen, Y., Li, J., et al. (2020). Clinical features and short-term outcomes of 221 patients with COVID-19 in Wuhan, China. Journal of Clinical 10 Virology 127, 104364. doi:10.1016/j.jcv.2020.104364

Zhao, M., Wang, M., Zhang, J., Gu, J., Zhang, P., Xu, Y., et al. (2020). Comparison of clinical characteristics and outcomes of patients with coronavirus disease 2019 at different ages. Aging (Albany NY) 12, 10070-10086. doi:10.18632/aging.103298

Zhou, F., Yu, T., Du, R., Fan, G., Liu, Y., Liu, Z., et al. (2020). Clinical course and risk factors 15 for mortality of adult inpatients with COVID-19 in Wuhan, China: a retrospective cohort study. The lancet 395, 1054-1062. doi:10.1016/S0140-6736(20)30566-3 